\title{
Management of Dry Eye in Sjögren's Syndrome
}

Michelle Hessen, $O D$

Sezen Karakus, MD

Esen Karamursel Akpek, MD*

\author{
Address \\ *Johns Hopkins Wilmer Eye Institute, 600 N. Wolfe Street, Baltimore, MD 21287, \\ USA \\ Email: esakpek@jhmi.edu
}

Published online: 16 July 2015

(C) Springer International Publishing AG 2015

This article is part of the Topical Collection on Sjögren's Syndrome

Keywords Dry eye $\cdot$ Sjögren's syndrome

\section{Opinion statement}

The treatment of dry eye in Sjögren's syndrome varies based on the severity of the disease. First-line therapy usually includes environmental changes and lubrication of the ocular surface with the use of artificial tears and/or ointments. Punctal occlusion may also help by extending available lubrication via natural or artificial tears. However, the occlusion of the puncta is usually recommended after ocular inflammation is addressed by topical medications. The main antiinflammatory topical treatment for Sjögren's syndrome-related dry eye is topical cyclosporine A. Cyclosporine A $0.05 \%$ is the only commercially available ophthalmic emulsion approved by the US Food and Drug Administration to be used in the treatment of dry eye. In severe cases, a $1 \%$ compounded preparation of the cyclosporine or more frequent instillation of $0.05 \%$ cyclosporine may be more efficient. Topical steroids can be considered as a short-term "pulse treatment" for inflammatory exacerbations of the disease given the high risk of complications with chronic use. In refractory cases, other anti-inflammatory treatments including autologous serum and topical tacrolimus may be considered. Contact lenses may help with the healing of the superficial keratitis or sterile ulcers. For the proper management of Sjögren's syndrome-related dry eye, it is important to diagnose the disease in its early stages and start topical or local antiinflammatory treatment before irreversible damage occurs in the lacrimal glands and on the ocular surface. The ocular surface complications of such as sterile corneal ulcers, opacification, and perforation may necessitate extensive surgeries, including corneal transplantation or keratoprosthesis implantation for the restoration of vision. 


\section{Introduction}

Dry eye is a common ocular condition encountered by primary eye care practitioners. An estimated $25 \%$ of patients presenting to general ophthalmology clinics complain of dry eye symptoms [1]. The most recent International Dry Eye Workshop (DEWS) redefined dry eye as "a multifactorial disease of the tears and ocular surface that results in symptoms of discomfort, visual disturbance, and tear film instability with potential damage to the ocular surface. It is accompanied by increased osmolarity of the tear film and inflammation of the ocular surface" [2]. The etiopathogenetic classification suggested by the DEWS distinguishes two main categories of dry eye according to which layer or component of the tear film is affected: aqueous tear deficiency and evaporative dry eye. Aqueous deficient dry eye is also further divided into two main groups: Sjögren's syndrome (SS)-related dry eye and non-SS dry eye.

SS is a chronic inflammatory disorder characterized by immune-mediated destruction of the salivary and lacrimal glands, with subsequent development of dry mouth and dry eye. SS may be seen as a primary disorder or as secondary to other well- defined autoimmune diseases, such as rheumatoid arthritis, systemic lupus erythematosus, systemic sclerosis, and polymyositis [2].

Several previous studies demonstrate that approximately one in ten patients with clinically significant dry eye have underlying SS [3]. Unfortunately, SS is greatly underrecognized in clinical practice, mostly due to diverse symptomatic expressions that make the initial diagnosis difficult. Dry eye in SS patients may result in various clinical manifestations including sterile corneal necrosis, vascularization, opacification, and even corneal perforation [4-6].

Early diagnosis of SS is important to manage the disease adequately as dry eye in SS is more severe and may require more aggressive treatment.

Current treatment of SS-related dry eye includes environmental changes, lubrication of the ocular surface either via replacement or conservation of tears, pharmacological treatment mainly targeting the associated ocular surface inflammation, and surgical procedures. The choice of therapy depends on the severity level of the dry eye findings.

\section{Treatment}

Currently, there are only three published treatment algorithms based primarily on grading the severity of symptoms and signs rather than on outcomes of therapy $[2,7,8 \bullet]$. One problem in assessing treatment outcomes is that most available publications on the treatment of dry eye group all causes of dry eye together as a single entity, although they might include various clinical entities with different pathogeneses, natural histories, severities, and outcomes.

As it is widely recognized that inflammation has a significant role in the etiopathogenesis of dry eye as well as the promotion of ocular surface disruption and symptoms of irritation, a number of anti-inflammatory treatments are currently in use for its management [9]. Many more anti-inflammatory medications are in the development or clinical trial phase. These agents inhibit the expression of inflammatory mediators on the ocular surface, thereby restoring the secretion of a healthy tear film and reducing the signs and symptoms of afflicted patients.

\section{Environmental changes}

Discussion with patients regarding home, workplace, and other environmental factors that can have an impact on dry eye symptoms may be beneficial. The patient should be cautioned to avoid prescription and nonprescription 
medications that have been associated with causing dry eye, such as analgesics, anticholinergics, antidepressants, antihistamines, diuretics, and many others. Dry indoor environments, windy and low-humidity outdoor conditions, and environments with excessive pollutants including smoke may exacerbate dry eye symptoms. Frequent, short breaks should be encouraged during extended computer use due to the associated reduced blink rate. The use of humidifiers is helpful in bedrooms and other rooms in which the patient spends a lot of time. Moisture-conserving eyewear such as spectacles with side shields, wraparound glasses, or goggles may be useful for patients to utilize to reduce tear evaporation.

\section{Topical treatments}

\section{Lubricants}

The initial treatment dry eye includes artificial tears as well as ointments. Preservative-free formulations are recommended especially if dosing more than four times per day is necessary. Preservative-containing eye drops, particularly those with benzalkonium chloride, are considered cytotoxic for ocular surface epithelium (including conjunctival goblet cells). Phosphate-containing eye drops should be used cautiously as they can promote corneal calcifications especially in the cases of corneal epithelial defects (corneal erosions or ulcers) [10]. Patients requiring frequent topical artificial tears should be placed on thicker formulations such as gels or ointments.

\section{Cyclosporine A}

Commercially available topical cyclosporine $0.05 \%$ (Restasis; Allergan, Irvine, CA) or $1 \%$ compounded preparation is used frequently for the treatment of various inflammatory ocular surface disorders [11]. Dosing topical cyclosporine at a frequency greater than twice a day may be more effective for patients who do not demonstrate improvement of severe dry eye disease with the twice-daily regimen $[12,13]$. The reduction in inflammation via inhibition of $\mathrm{T}$ cell activation and downregulation of inflammatory cytokines in the conjunctiva and lacrimal gland $[14,15]$ is thus thought to allow enhanced tear production [16-18]. Topical cyclosporine also increases goblet cell density and decreases epithelial cell apoptosis [19].

The efficacy of $0.05 \%$ cyclosporine A was evaluated in 26 patients with dry eye due to primary and secondary SS [20•]. All subjective symptoms (burning and pricking sensation, light sensitivity, and pain) and objective signs (Schirmer test, tear break-up time [TBUT], and redness) were statistically significantly improved after 1-week and 1-month follow-up examinations ( $p=0.0001)$. Compared with a control group of 20 patients using saline drops, there was significant improvement in all parameters. Researchers concluded that topical $0.05 \%$ cyclosporine $A$ is an effective treatment option for dry eye due to SS after a 1-month follow-up period.

\section{Corticosteroids}

Through several mechanisms of action, topical steroids help reduce ocular inflammation. Corticosteroids function via suppression of cellular infiltration, 
capillary dilation, proliferation of fibroblasts, and collagen deposition. They stabilize intracellular and extracellular membranes. Corticosteroids increase the synthesis of lipocortins that block phospholipase $\mathrm{A}_{2}$ and inhibit histamine synthesis in the mast cells [21]. Inhibition of phospholipase $A_{2}$, an essential step in the inflammatory cascade, prevents the conversion of phospholipids to arachidonic acid. Corticosteroids also interfere with transcription factor NF-kB, which regulates synthesis of a number of proinflammatory molecules, thereby stimulating lymphocyte apoptosis.

Several clinical studies have demonstrated the effectiveness of topical steroids for the treatment of dry eye. In a retrospective clinical series, topical administration of a $1 \%$ solution of nonpreserved methylprednisolone given three or four times daily for several weeks to patients with SS-related dry eye provided moderate to complete relief of symptoms in all patients [22]. In addition, there was a decrease in corneal fluorescein staining score and complete resolution of filamentary keratitis. This therapy was effective even for patients suffering from severe dry eye who had no improvement from maximum aqueous tear enhancement/replacement therapies.

In a prospective study of 53 patients with SS, the long-term recurrence rate of dry eye symptoms and signs after short-term pulse topical preservative-free methylprednisolone therapy was evaluated [23]. Therapy was started at 4 times a day for the first 2 weeks and tapered off every 2 weeks until complete discontinuation. Improvement in subjective symptoms and corneal staining scores occurred as early as 2 weeks. Significantly improved TBUT and Schirmer test results were observed at the end of the treatment period. Impression cytology revealed a significantly increased number of conjunctival goblet cells. After the first pulse therapy, mean drug-free remission time was 56.6 weeks; 11 patients $(20.8 \%)$ had a recurrence of symptoms and signs. After the second pulse therapy, mean drug-free remission time was 72.4 weeks; only 1 patient had a recurrence. No serious complications, including intraocular pressure elevation and cataract formation, occurred during the entire follow-up period. Patients treated with topical corticosteroids should be monitored closely for known risks of cataract formation, glaucoma, corneal thinning, and infectious keratitis [24].

\section{Nonsteroidal anti-inflammatory drugs}

Nonsteroidal anti-inflammatory drugs (NSAIDs) are utilized in the management of many ocular conditions including allergic conjunctivitis, postoperative ocular pain, cystoid macular edema status postcataract surgery, and dry eye. The mechanism by which NSAIDs treat inflammation is by inhibiting the production of prostaglandins via the cyclooxygenase enzyme [25]. NSAIDs, specifically diclofenac, have been shown to reduce corneal sensitivity [26]. This may bring an adjunctive insult to disrupted epithelium in patients with dry eye. Several cases of corneal melt have been described in the literature associated with use of topical NSAIDs, including the use of diclofenac [27, 28], ketorolac [29], nepafenac [30], and bronfenac [31-33]. In all of these cases, preexisting epitheliopathy was identified. Although the exact relationship between corneal melt and topical NSAIDs use is still not clear, various suggested mechanisms include activation of matrix metalloproteinases (MMPs), impairment of wound healing, and a neurotrophic effect resulting from analgesic action of these drugs 
[27]. Short-term use of NSAIDs can help to ameliorate symptoms of ocular discomfort in dry eye. However, they should not be used in SS-related dry eye due to the possible corneal side effects.

\section{Autologous serum}

Serum contains several anti-inflammatory factors that have the capability to inhibit soluble mediators of the ocular surface inflammatory cascade of dry eye. These include inhibitors of inflammatory cytokines (e.g., interleukin [IL]-1 RA and soluble tumor necrosis factor [TNF]-receptors) and MMP inhibitors (e.g., TIMPs) [34-36]. Clinical trials have shown autologous serum drops to improve ocular irritation symptoms and conjunctival and corneal dye staining in dry eye that occurs in the setting of SS [37-39]. Conversely, there is greater risk of microbial growth as autologous serum drops, in addition to antimicrobial agents, contains a high protein content, and is generally nonpreserved [40].

Recent studies have investigated cord serum drops (prepared from donor umbilical cord serum) $[41,42]$ as well as allogenic serum drops from a relative donor. A clinical trial of 17 patients with graft-versus-host-disease and 13 patients with SS-associated dry eye was performed for 1 month with cord blood serum. Patients received cord blood once a day (containing 0.15-ng epithelial growth factor per drop). Patients reported a decrease in discomfort symptoms as measured by the Ocular Surface Disease Index (OSDI) score. Clinical findings such as impression cytology score, tear osmolarity, and corneal sensation (measured with a Cochet-Bonnet esthesiometer) improved significantly [42].

A randomized, double-blind study of 26 patients (51 eyes) with SS was performed to compare autologous serum diluted with sodium hyaluronate and saline solution [43•]. Patients underwent a complete ophthalmic assessment including tear film evaluation and corneal and conjunctival impression cytology at the beginning of the study and 2 months later. Autologous serum diluted with sodium hyaluronate caused a significant improvement of the tear film stability, fluorescein and rose bengal stain, break-up time, corneal and conjunctival squamous metaplasia, as well as in patient subjective perception. Preparations diluted with sodium hyaluronate were better tolerated by patients and required a lower number of drop administrations due to the gradual release of growth factor, which increased their duration and effect on the ocular surface.

In a nonrandomized clinical trial comparing two types of topical serum, seven SS patients were treated with autologous serum, and ten SS patients were treated with umbilical cord serum for 2 months [44]. At the end of the treatment period, both groups showed significant improvements in symptom scores, TBUT, corneal staining score, grade of squamous metaplasia, and goblet cell density. The group receiving umbilical cord serum showed greater improvement in symptom scores and corneal staining scores at 1 and 2 months after treatment compared with the group receiving autologous serum. Goblet cell density scores were significantly higher in the umbilical cord serum group compared with autologous serum group at 2 months as well.

\section{Tacrolimus}

Topical tacrolimus, available in 0.03 and $0.1 \%$ as an ointment as well as compounded eye drops, is promising for the treatment of dry eye in the setting of SS. A prospective double-blind randomized study was performed to evaluate 
clinical efficacy of $0.03 \%$ tacrolimus in 48 eyes of 24 patients with dry eye related to Sjögren syndrome treatment [ $45 \bullet$. The patients were instructed to use the eye drops every $12 \mathrm{~h}$ : the tacrolimus group received $0.03 \%$ tacrolimus (almond oil as vehicle) and the other group received the almond oil vehicle. After 7 days of treatment, the average fluorescein and Rose Bengal scores improved statistically, and even more after 90 days. Schirmer I and break-up time showed improvement relative to baseline after 28 days of treatment, however were unchanged after $7,14,28$, and 90 days. There was no statistically significant difference in Schirmer I, break-up-time, fluorescein, and Rose Bengal staining in the vehicle group.

Systemic tacrolimus has been reported to be effective for improving dry eye associated with inflammatory systemic conditions; however, there are potential adverse reactions to be aware of when administering long-term systemic therapy [46]. This topical anti-inflammatory agent (previously known as FK506) is a macrolide antibiotic isolated from Streptomyces tsukubaensis fermentation [47]. Although the mechanism of tacrolimus is similar to cyclosporine A, the potency in vitro has been shown to be significantly greater, exhibiting similar effects at 100 times lower concentrations [48]. Only when bound to immunophilin does it become biologically active, thus effectively inhibiting calcineurin and inhibiting T and B lymphocyte activation via reduction in IL-2 synthesis [4955]. Tacrolimus suppresses the immune response by inhibiting the release of other inflammatory cytokines as well (e.g., IL-3, IL-4, IL-5, IL-8, interferon[gamma], and TNF-[alpha]) [56-59].

\section{Diquafosol (phase III clinical trials in the USA; approved for use in Japan)}

Diquafosol is a P2Y2 agonist (a purinergic stimulator) and topical secretagogue for chloride ions that increases aqueous tear volume in animal models by directly transporting fluid across the serosal membranes of the ocular surface and may also stimulate mucin secretion [60]. In a randomized, double-masked, parallel-group trial of 527 dry eye subjects, 24 weeks of treatment with 2 concentrations of diquafosol ( 1 and $2 \%$ ) versus placebo instilled 4 times daily were compared [60]. Subjects treated with $2 \%$ diquafosol had significantly lower corneal staining scores compared with placebo at the 6-week primary efficacy time point $(p<0.001)$, and superiority continued throughout the 24-week study. Results for conjunctival staining were consistent with those observed for corneal staining. Schirmer scores at week 6 were significantly higher with diquafosol treatment than with placebo $(p<0.030$ or $p=0.030$ ). Diquafosol has a favorable risk/benefit profile in a broad spectrum of patients with dry eye disease and is a novel topical treatment of dry eye.

\section{Systemic treatments}

\section{Secretagogues}

Acetylcholine acting through muscarinic receptors controls exocrine gland secretion, including lacrimation. Patients with SS have serum autoantibodies that bind to $\mathrm{M} 3$ receptors. Lymphocytic cell infiltration and glandular destruction 
are thought to be the main mechanism of dryness associated with SS; however, the M3 receptor-binding autoantibodies may also contribute to the pathogenesis [61]. Cevimeline and Pilocarpine are examples of secretagogues for SS dry eye, as discussed below.

Cevimeline hydrochloride (Evoxac, Daiichi Pharmaceutical, Montvale, NJ), administered as $30 \mathrm{mg}$ tablets taken orally 3 times daily, is a derivative of acetylcholine that binds to M3 receptors in exocrine glands and increases exocrine gland secretion. In randomized prospective double-blinded trials to evaluate the efficacy of cevimeline in dry eye, the cevimeline treatment arm had improved tear biometrics, as measured by Schirmer testing, Rose Bengal and fluorescein staining, and TBUT, as well as subjective patient symptom reporting [62-64]. Although promising in terms of efficacy, systemic cevimeline has been associated with side effects of nausea, abdominal pain, sweating, headache, dizziness, and cardiac arrhythmias, resulting in a withdrawal rate of $14-19 \%$ from these clinical trials $[63,64]$.

Pilocarpine (Salagen tablets, Novartis Pharmaceuticals, Basel, Switzerland) is a muscarinic cholinergic parasympathomimetic agonist that binds to M3 receptors and stimulates exocrine glands. Salagen is currently available in 5and 7.5-mg tablets administered orally. Most patients require $20 \mathrm{mg} /$ daily in divided doses for therapeutic response. In a multicenter, placebo-controlled, fixed dose trial, 373 patients with primary or secondary SS and clinically significant dry mouth and dry eyes were randomized to receive 10 or $20 \mathrm{mg} /$ day of pilocarpine or placebo tablets for 12 weeks [65]. For global improvement of dry eye symptoms, a significant benefit was observed in the 20-mg pilocarpine group versus the placebo group. The 10-mg pilocarpine group had no better symptomatic relief of oral or ocular symptoms than the placebo group. Additionally, a greater proportion of patients taking 20-mg pilocarpine tablets showed clinically significant improvement (compared with those taking placebo) in the ability to focus their eyes while reading and reduced severity of blurred vision. Excessive sweating was the most commonly reported side effect, occurring in more than $40 \%$ of patients. In a multicenter, randomized, placebo-controlled, dose-adjustment study involving 256 patients with SS, the safety and efficacy of oral pilocarpine (20 or $30 \mathrm{mg} /$ daily) for relief of dry mouth and dry eye symptoms were assessed over 12 weeks [66]. There was significant symptomatic improvement of dry eye at 12 weeks with pilocarpine $20 \mathrm{mg} /$ daily. Of note, the use of artificial tears also decreased significantly in the pilocarpine group at the end of the study period.

\section{Hydroxychloroquine}

Hydroxychloroquine (HCQ) has shown mixed results for the treatment of SS-associated dry eye. The available results include a study showing improvement in symptoms and objective measures of dryness and 
corneal surface findings in 50 to $60 \%$ of 40 patients who received HCQ ( 6 to $7 \mathrm{mg} / \mathrm{kg} /$ day) for 24 to 48 months [67]; a study of 32 patients treated for at least 48 months with HCQ who were found to have significant worsening in dry eye symptoms and objective measures of dryness and corneal epithelial findings 3 months following discontinuation of therapy [68]; a small series of 21 patients showing nonsignificant benefit in dry eye findings but significant benefit for dry mouth [69]; and a randomized 2-year crossover trial involving only 19 patients that failed to demonstrate any significant benefit in ocular symptoms or findings, despite some improvement in signs of inflammation including acute phase reactants and hyperglobulinemia [70]. In a randomized trial of HCQ in 120 patients, patient-reported dryness symptoms (assessed by a visual analog scale) and Schirmer test results did not show a significant improvement from baseline when compared to placebo at 24 weeks of treatment [71].

\section{Rituximab}

Rituximab is a monoclonal antibody directed against CD20 that targets B cells. There are limited reports of benefit for dry eye (or dry mouth), despite evidence of benefit for systemic and extraglandular manifestations of SS. Some benefit was suggested in a randomized trial involving 30 patients in only one of three measures of lacrimal gland function (corneal staining with lissamine green but not Schirmer's testing or TBUT) and statistically significant improvement in subjective scoring of ocular dryness compared with baseline values [72]. In another randomized trial involving 17 patients, there was no significant change in glandular function 6 months after administration of rituximab, and there was no improvement in Schirmer's test scores or in the unstimulated salivary flow rate at that time point [73]. In a prospective, two-center follow-up study of 41 patients with early active SS, Schirmer test results were significantly improved at 120 weeks in the 19 who received rituximab treatments every 24 weeks, relative to the 22 who received conventional disease-modifying antirheumatic drug therapy [74]. These findings need to be reproduced in a randomized trial but suggest that prolonged therapy with rituximab in early active disease is needed to realize significant clinical benefit.

\section{Procedures and surgical treatment}

\section{Punctal occlusion}

Minimizing tear drainage through use of punctal plugs as well as thermal cauterization may contribute to healing of the ocular surface by extending available lubrication via natural or artificial tears. Recurrent loss of punctal plugs could be problematic, often limiting the effectiveness of this treatment [75]. It is generally recommended to address the ocular surface inflammation by topical medications prior to the consideration of the punctal occlusion. 
A prospective, randomized study was performed to compare the effects of treatment with punctal plugs versus artificial tears on visual function for primary SS with dry eye [76•]. Forty-two eyes of 42 patients with primary SS were enrolled and allocated randomly into artificial tears (AT) group and punctal plugs (PP) group. The OSDI was used along with fluorescein staining for TBUT, the Schirmer test I, and contrast sensitivity. These measures were performed before treatment and repeated 3 months after treatment. A follow-up of 3 months was achieved in 40 eyes of 40 patients, including 19 eyes in the AT group and 21 eyes in the PP group. Statistically significant improvements were observed in the OSDI scores and corneal fluorescein staining scores, and BUT after treatment compared to those of pretreatment. The values of Schirmer test I and TBUT in the PP group were significantly more improved than those in the AT group. The medium- and high-level frequency contrast sensitivities were greatly improved in simulated daylight, night, and glare disability conditions after treatment with artificial tears and punctal plugs. However, the changes in contrast sensitivity did not significantly differ between groups. Both AT and PP relieved dry eye symptoms, repaired corneal lesions, enhanced tear film stability, and improved contrast sensitivity. Plugs could improve tear film stability and elongate the tear break-up time better than AT.

\section{Contact lenses}

With the evolution of silicone hydrogel materials for soft contact lenses providing high oxygen permeability, extended wear modality has become an acceptable option for assisting with ocular surface repair. Therapeutic bandage contact lenses provide protection of the cornea from the mechanical friction of the eyelids as well as exposure to the external environment and evaporation of the tear film. However, the risk of infection is considerable for longer term use.

Rigid gas permeable scleral lenses create a tear reservoir for constant lubrication of the corneal surface while maintaining necessary oxygen supply. These lenses are able to mask surface irregularities that also contribute to improvement in vision. Results of a retrospective chart review of 49 consecutive patients (76 eyes) with ocular surface disease at the setting of various systemic conditions, including SS, showed improvement in visual acuity, healing of epithelial defects, reduction in photophobia, and improvement in quality of life [77].

\section{Surgical treatment}

For severe dry eye cases, when the traditional treatments are not satisfactory, major salivary gland transplantation as a surgical procedure may be considered. Autologous submandibular gland (SMG) transplantation into the temporal fossa with implantation of Wharton's duct into the upper conjunctival fornix has been found effective for severe dry eye cases [78-80]. Clinical studies have shown that the tear-like, seromucinous secretion from the transplant tissue reduced the symptoms and the frequency of use of the lubricants and also improved clinical signs such as TBUT and corneal staining [79, 80]. Although some cases of hypersecretion have been reported postoperatively, partial transplantation of SMG may prevent or reduce this undesirable complication [81]. 


\section{Conflict of Interest}

Michelle Hessen, Sezen Karakus, and Esen Karamursel Akpek declare that they have no conflict of interest.

\section{Human and Animal Rights and Informed Consent}

This article does not contain any studies with human or animal subjects performed by any of the authors.

\section{References and Recommended Reading}

Papers of particular interest, published recently, have been

highlighted as:

- Of importance

1. Doughty MJ, Fonn D, Richter D, et al. A patient questionnaire approach to estimating the prevalence of dry eye symptoms in patients presenting to optometric practices across Canada. Optom Vis Sci. 1997;74:62431 .

2. The definition and classification of dry eye disease: report of the Definition and Classification Subcommittee of the International Dry Eye WorkShop (2007). Ocul Surf. 2007;5(2):75-92. Review

3. Akpek EK, Klimava A, Thorne JE, et al. Evaluation of patients with dry eye for presence of underlying Sjögren syndrome. Cornea. 2009;28:493-7.

4. Kassan SS, Moutsopoulos HM. Clinical manifestations and early diagnosis of Sjögren syndrome. Arch Intern Med. 2004;164(12):1275-84.

5. Gottsch JD, Akpek EK. Topical cyclosporin stimulates neovascularization in resolving sterile rheumatoid central corneal ulcers. Trans Am Ophthalmol Soc. 2000;98:81-7. discussion 87-90.

6. Ou JI, Manche EE. Corneal perforation after conductive keratoplasty in a patient with previously undiagnosed Sjögren syndrome. Arch Ophthalmol. 2007;125:11312.

7. Behrens A, Doyle JJ, Stern L, et al. Dysfunctional Tear Syndrome Study Group. Dysfunctional tear syndrome: a Delphi approach to treatment recommendations. Cornea. 2006;25:900-7.

8. Foulks GN, Forstot SL, Donshik PC, et al. Clinical guidelines for management of dry eye associated with Sjogrens disease. Ocul Surf. 2015;12(2):118-32.

Tear supplementation and stabilization, control of inflammation of the lacrimal glands and ocular surface, and possible stimulation of tear production are treatment options that are used according to the character and severity of dry eye disease.

9. Hessen M, Akepk EK. Dry Eye: An inflammatory ocular disease. J Ophthalmic Vis Res. 2014;9(2):240-50.

10. Bernauer W, Thiel MA, Kurrer M, et al. Corneal calcifications following intensified treatment with sodium hyaluronate artificial tears. Br J Ophthalmol. 2006;90:285-8.
11. Utine CA, Stern M, Akpek EK. Clinical review: topical ophthalmic use of cyclosporin A. Ocul Immunol Inflamm. 2010;18:352-61.

12. Gupta A, Sadeghi PB, Akpek EK. Occult thyroid eye disease in patients presenting with dry eye symptoms. Am J Ophthalmol. 2009;147:919-23.

13. Dastjerdi MH, Hamrah P, Dana R. High frequency topical cyclosporine $0.05 \%$ in the treatment of severe dry eye refractory to twice-daily regimen. Cornea. 2009;28:1091-6.

14. Pflugfelder SC, Wilhelmus KR, Osato MS, et al. The auto-immune nature of aqueous tear deficiency. Ophthalmology. 1986;93:1513-7.

15. Stern ME, Gao J, Siemasko KF, et al. The role of the lacrimal gland functional unit in the pathophysiology of dry eye. Exp Eye Res. 2004;78:40916.

16. Stevenson D, Tauber J, Reis BL. Efficacy and safety of cyclosporine A ophthalmic emulsion in the treatment of moderate to severe dry eye disease: a dose-ranging, randomized trial. The Cyclosporine A Phase 2 Study Group. Ophthalmology. 2000;107:967-74.

17. Sall K, Stevenson OD, Mundorf TK, et al. Two multicenter, randomized studies of the efficacy and safety of cyclosporine ophthalmic emulsion in moderate to severe dry eye disease. CsA Phase 3 Study Group. Ophthalmology. 2000;107:631-9.

18. Laibovitz RA, Solch S, Andriano K, et al. Pilot trial of cyclosporine $1 \%$ ophthalmic ointment in the treatment of keratoconjunctivitis sicca. Cornea. 1993;12:315-23.

19. Kunert KS, Tisdale AS, Gipson IK. Goblet cell numbers and epithelial proliferation in the conjunctiva of patients with dry eye syndrome treated with cyclosporine. Arch Ophthalmol. 2002;120:330-7.

20. Devec1 H, Kobak S. The efficacy of topical $0.05 \%$ cyclosporine A in patients with dry eye disease associated with Sjögren's syndrome. Int Ophthalmol. 2015;34:1043-8. 
This prospective study concluded topical $0.05 \%$ cyclosporine A is an effective treatment option for keratoconjunctivitis sicca due to SS after a 1-month follow-up period.

21. Comstock T, DeCory H. Advances in corticosteroid therapy for ocular inflammation: loteprednol etabonate. Int J Inflamm. 2012;2012:789623.

22. Marsh P, Pflugfelder SC. Topical non-preserved methylpresdnisolone therapy of keratoconjunctivits sicca in Sjogren's syndrome. Ophthalmology. 1999; 106:811-6.

23. Hong S, Kim T, Chung SH, et al. Recurrence after topical nonpreserved methylprednisolone therapy for keratoconjunctivitis sicca in Sjögren's syndrome. J Ocul Pharmacol Ther. 2007;23:78-82.

24. McGhee CN, Dean S, Danesh-Meyer H. Locally administered ocular corticosteroids: benefits and risks. Drug Saf. 2002;25(1):33-55.

25. Vane JR, Bakhle YS, Botting RM. Cyclooxygenases 1 and 2. Annu Rev Pharmacol Toxicol. 1998;38:97-120.

26. Aragona P, Tripodi G, Spinella R, et al. The effects of the topical administration of non-steroidal anti-inflammatory drugs on corneal epithelium and corneal sensitivity in normal subjects. Eye. 2000;14:206-10.

27. Gokhale NS, Vemuganti GK. Diclofenac-induced acute corneal melt after collagen crosslinking for

keratoconus. Cornea. 2010;29:117-9.

28. Flach AJ. Corneal melts associated with topically applied nonsteroidal anti-inflammatory drugs. Trans Am Ophthalmol Soc. 2001;99:205-12.

29. Khalifa YM, Mifflin MD. Keratitis and corneal melt with ketorolac tromethamine after conductive keratoplasty. Cornea. 2011;30:477-8.

30. Di Pascuale MA, Whitson JT, Mootha VV. Corneal melting after use of nepafenac in a patient with chronic cystoid macular edema after cataract surgery. Eye Contact Lens. 2008;34:129-30.

31. Asai T, Nakagami T, Mochizuki M, et al. Three cases of corneal melting after instillation of a new nonsteroidal anti-inflammatory drug. Cornea. 2006;25:224-7.

32. Isawi H, Dhaliwal DK. Corneal melting and perforation in Stevens Johnson syndrome following topical bromfenac use. J Cataract Refract Surg. 2007;33:1644-6.

33. Prasher P. Acute corneal melt associated with topical bromfenac use. Eye Contact Lens. 2012;38:260-2.

34. Liou LB. Serum and in vitro production of IL-1 receptor antagonist correlate with C-reactive protein levels in newly diagnosed, untreated lupus patietns. Clin Exp Rheumatol. 2001;19:515-23.

35. Ji H, Pettit A, Ohmura K, et al. Critical roles for interleukin 1 and tumor necrosis factor alpha in antibodyinduced arthritis. J Exp Med. 2002;196:77-85.

36. Paramo JA, Orbe J, Fernandez J. Fibrinolysis/ proteolysis balance instable angina pectoris in relation to angiographic findings. Thromb Haemost. 2001;86:636-9.

37. Fox RI, Chan R, Michelson JB, et al. Beneficial effect on artificial tears made with autologous serum in patients with keratoconjunctivitis sicca. Arthritis Rheum. 1984;27:459-61.
38. Kono I, Kono K, Narushima K, et al. Beneficial effect of the local application of plasma fibronectin and autologous serum in patients with keratoconjunctivitis sicca of Sjogren's syndrome. Ryumachi. 1986;26:339-43.

39. Tsubota K, Goto E, Fujita H, et al. Treatment of dry eye by autologous serum application in Sjogren's syndrome. Br J Ophthamol. 1999;83:390-5.

40. Tananuvat N, Daniell M, Sullivan LJ, et al. Controlled study of the use of autologous serum in dry eye patients. Cornea. 2001;20:802-6.

41. Versura P, Profazio V, Buzzi M, et al. Efficacy in standardized and quality-controlled cord blood serum eye drop therapy in the healing of severe corneal epithelial damage in dry eye. Cornea. 2013;32:412-8.

42. Yoon KC, Jeong IY, Im SK, et al. Therapeutic effect of umbilical cord serum for the treatment of dry eye associated with graft-versus-host disease. Bone Marrow Transplant. 2007;39:231-5.

43. López-García JS, García-Lozano I, Rivas L, et al. Autologous serum eye drops diluted with sodium hyaluronate: clinical and experimental comparative study. Acta Ophthalmol. 2014;92(1):e22-9.

Sodium hyaluronate is an excellent vehicle for diluting autologous serum due to the gradual release of growth factor, increasing the serum's duration and effect on the ocular surface. Preparations diluted with sodium hyaluronate are better tolerated by patients and require a lower number of drops administrations compared to saline.

44. Yoon KC, Heo H, Im SK, et al. Comparison of autologous serum and umbilical cord serum eye drops for dry eye syndrome. Am J Ophthalmol. 2007;144:86-92.

45. Moscovici B, Holzchuh R, Sakasseqawa-Naves FE, et al. Treatment of Sjogren's syndrome dry eye using 0.03\% tacrolimus eye drop: Prospective double-blind randomized study. Cont Lens Anterior Eye. 2015;S13670484 .

Topical $0.03 \%$ tacrolimus eye drops successfully improved tear stability and ocular surface status in patients with SS-related dry eyes.

46. Thomson AW, Bonham CA, Zeevi A. Mode of action of tacrolimus (FK506): molecular and cellular mechanisms. Ther Drug Monit. 1995; 17:584-91.

47. Kino T, Hatanaka H, Miyata S, et al. FK-506, a novel immunosuppressant isolated from Streptomyces. I. Fermentation isolation, and physio-chemical and biological characteristics. J Antibiot (Tokyo). 1987;40:1249-55.

48. Attas-Fox L, Barkana Y, Iskhakov V, et al. Topical tacrolimus $0.03 \%$ ointment for intractable allergic conjunctivitis: an open-label pilot study. Curr Eye Res. 2008;33:545-9.

49. Bertelmann E, Pleyer U. Immunomodulatory therapy in ophthalmology-is there a place for topical application? Ophthalmologica. 2004;218:359-67.

50. Fei WL, Chen JQ, Yuan J, et al. Preliminary study of the effect of FK506 nanospheric-suspension eye drops on 
rejection of penetrating keratoplasty. J Ocul Pharmacol Ther. 2008;24:235-44.

51. Fujita E, Teramura Y, Mitsugi K, et al. Absorption, distribution, and excretion of 14C-labeled tacrolimus (FK506) after a single or repeated ocular instillation in rabbits. J Ocul Pharmacol Ther. 2008;24:333-43.

52. Fujita E, Teramura Y, Shiraga T, et al. Pharmacokinetics and tissue distribution of tacrolimus (FK506) after a single or repeated ocular instillation in rabbits. J Ocul Pharmacol Ther. 2008;24:309-19.

53. Nishino K, Fukushima A, Okamoto $S$, et al. Suppression of experimental immune-mediated blepharoconjunctivitis in Brown Norway rats by topical application of FK506. Graefes Arch Clin Exp Ophthalmol. 2002;240:137-43.

54. Pleyer U, Lutz S, Jusko WJ, et al. Ocular absorption of topically applied FK506 from liposomal and oil formulations in the rabbit eye. Invest Ophthalmol Vis Sci. 1993;34:2737-42.

55. Sasakawa Y, Sakuma S, Higashi Y, et al. FK506 suppresses neutrophil chemoattractant production by peripheral blood mononuclear cells. Eur J Pharmacol. 2000;403:281-8.

56. Reitamo S, Van Leent EJ, Ho V, et al. Efficacy and safety of tacrolimus ointment compared with that of hydrocortisone acetate ointment in children with atopic dermatitis. J Allergy Clin Immunol. 2002;109:539-46.

57. Reitamo S, Rustin M, Ruzicka T, et al. Efficacy and safety of tacrolimus ointment compared with that of hydrocortisone butyrate ointment in adult patients with atopic dermatitis. J Allergy Clin Immunol. 2002;109:547-55.

58. Reitamo S, Remitz A, Kyllönen H, et al. Topical noncorticosteroid immunomodulation in the treatment of atopic dermatitis. Am J Clin Dermatol. 2002;3:381-8.

59. Aoki S, Mizote H, Minamoto A, et al. Systemic FK506 improved tear secretion in dry eye associated with chronic graft versus host disease. Br J Ophthalmol. 2005;89(2):243-4.

60. Tauber J, Davitt WF, Bokosky JE, et al. Double-masked, placebo-controlled safety and efficacy trial of diquafosol tetrasodium (INS365) ophthalmic solution for the treatment of dry eye. Cornea. 2004;23:784-92.

61. Akpek EK, Lindsley KB, Adyanthaya RS. Treatment of Sjogren's syndrome-associated dry eye: An evidencebased review. Ophthalmolology. 2011;118:1242-52.

62. Ono M, Takamura E, Shinozaki K, Tsumura T, Hamano $\mathrm{T}$, Yagi Y, et al. Therapeutic effect of cevimeline on dry eye in patients with Sjogren's syndrome: a randomized, double-blind clinical study. Am J Ophthalmol. 2004;138(1):6-17.

63. Fife RS, Chase WF, Dore RK, Wiesenhutter CW, Lockhart $\mathrm{PB}$, Tindall E, et al. Cevimeline for the treatment of xerostomia in patients with Sjogren syndrome: a randomized trial. Arch Intern Med. 2002;162:1293-300.

64. Petrone D, Condemi JJ, Fife R, Gluck O, Cohen S, Dalgin P. A double-blind, randomized, placebocontrolled study of cevimeline in Sjogren's syndrome patients with xerostomia and keratoconjunctivitis sicca. Arthritis Rhem. 2002;46:748-54.

65. Vivino FB, Al-Hashimi I, Khan Z, et al. P92-01 Study Group. Pilocarpine tablets for the treatment of dry mouth and dry eye symptoms in patients with Sjögren syndrome: a randomized, placebo-controlled, fixeddose, multicenter trial. Arch Intern Med. 1999;159:174-81.

66. Papas AS, Sherrer YS, Charney M, et al. Successful treatment of dry mouth and dry eye symptoms in Sjögren's syndrome patients with oral pilocarpine: a randomized, placebocontrolled, dose-adjustment study. J Clin Rheumatol. 2004;10:169-77.

67. Fox RI, Dixon R, Guarrasi V, Krubel S. Treatment of primary Sjögren's syndrome with hydroxychloroquine: a retrospective, open-label study. Lupus. 1996;5 Suppl 1:S31.

68. Yavuz S, Asfuroğlu E, Bicakcigil M, Toker E. Hydroxychloroquine improves dry eye symptoms of patients with primary Sjogren's syndrome. Rheumatol Int. 2011;31:1045-9.

69. Rihl M, Ulbricht K, Schmidt RE, Witte T. Treatment of sicca symptoms with hydroxychloroquine in patients with Sjogren's syndrome. Rheumatology (Oxford). 2009;48:796-9.

70. Kruize AA, Hené RJ, Kallenberg CG, et al. Hydroxychloroquine treatment for primary Sjögren's syndrome: a two year double blind crossover trial. Ann Rheum Dis. 1993;52:360-4.

71. Gottenberg JE, Ravaud P, Puéchal X, et al. Effects of hydroxychloroquine on symptomatic improvement in primary Sjögren syndrome: the JOQUER randomized clinical trial. JAMA. 2014;312:24958.

72. Meijer JM, Meiners PM, Vissink A, et al. Effectiveness of rituximab treatment in primary Sjögren's syndrome: a randomized, double-blind, placebo-controlled trial. Arthritis Rheum. 2010;62:960-8.

73. Dass S, Bowman SJ, Vital EM, et al. Reduction of fatigue in Sjögren syndrome with rituximab: results of a randomised, double-blind, placebocontrolled pilot study. Ann Rheum Dis. 2008;67:1541-4.

74. Carubbi F, Cipriani P, Marrelli A, et al. Efficacy and safety of rituximab treatment in early primary Sjögren's syndrome: a prospective, multi-center, follow-up study. Arthritis Res Ther. 2013;15:R172.

75. Ferrara JL, Levine JE, Reddy P, Holler E. Graft-versushost disease. Lancet. 2009;373:1550-61.

76.• Qiu W, Liu Z, Ao M, et al. Punctal plugs versus artificial tears for treating primary Sjögren's syndrome with keratoconjunctivitis SICCA: a comparative observation of their effects on visual function. Rheumatol Int. 2013;33:2543-8.

Both artificial tears and punctal plugs relieved dry eye symptoms, repaired corneal lesions, enhanced tear film stability, and improved contrast sensitivity. Punctal plugs could improve tear film stability and elongate the tear break-up time better than artificial tears. 
77. Romero-Rangel T, Stavroua P, Cotter J, et al. Gaspermeable scleral contact lens therapy in ocular surface disease. Am J Ophthalmol. 2000;130(1):25-32.

78. Geerling G, Sieg P, Bastian GO, Laqua H. Transplantation of the autologous submandibular gland for most severe cases of keratoconjunctivitis sicca. Ophthalmology. 1998;105:327-35.

79. Jacobsen HC, Hakim SG, Lauer I, et al. Long-term results of autologous submandibular gland transfer for the surgical treatment of severe keratoconjunctivitis sicca. J Craniomaxillofac Surg. 2008;36:227-33.

80. Borrelli M, Schröder C, Dart JK, et al. Long-term followup after submandibular gland transplantation in severe dry eyes secondary to cicatrizing conjunctivitis. Am J Ophthalmol. 2010;150:894-904.

81. Qin J, Zhang L, Cai ZG, et al. Microvascular autologous transplantation of partial submandibular gland for severe keratoconjunctivitis sicca. Br J Ophthalmol. 2013;97:1123-8. 